little room to doubt that the risk of developing bladder cancer is increased by exposure to certain aromatic amines in the laboratory. The careless use of benzidine in testing for occult blood and in spraying chromatographs are particular examples of where hazards may occur.

Studies on laboratory animals point to many other agents used in laboratories as possibly being carcinogenic in man, but it is difficult to pinpoint which of the suspect compounds are really hazardous. A chemical agent that gives rise to cancer of one type in one animal species may predispose to cancers of one or more entirely different types in another. Laboratory workers may handle a multitude of chemical compounds during the course of a working life-time, and a long and variable latent interval between start of exposure to a carcinogen and the first evidence of its effect renders the task of associating cause with effect difficult. The fact that exposure may cease long before cancer develops serves to magnify the difficulty further. In any case cancer of one type or another is such a common disease that when it occurs in a laboratory worker the possibility of its being occupational in origin is easy to overlook.

A report from the Epidemiology Branch of the National Cancer Institute in Bethesda ${ }^{2}$ should increase general awareness of carcinogenic hazards in laboratories. F. P. Li and his colleagues scrutinized 3,637 out of a possible 4,644 death certificates relating to members of the American Chemical Society who died between April 1948 and July 1967 and coded the causes of death according to the World Health Organization manual. ${ }^{3}$ They then compared frequencies of death from specific causes with expected frequencies based on the mortality of professional men in comparable age groups. ${ }^{4}$ Among male chemists of 20-64 years of age there were 444 deaths from cancer against 354 expected. Of the 90 excess cancer deaths 41 were attributable either to malignant lymphoma or to cancer of the pancreas. Cancer of one form or another was also commoner than expected as a cause of death in male chemists over the age of 64 (250 compared with 218). In this case, cancer of the pancreas or lymphoma accounted for 23 of the 42 excess deaths and cancers of the intestines or rectum for 12. Deaths from cancers of the lung and stomach, on the other hand, were less frequent in chemists than expected. $\mathrm{Li}$ and his colleagues did not find a significant excess of deaths from cancer of the urinary bladder, but the numbers of cases expected and found were small.

The authors list several reasons why their findings should be interpreted with caution. However, the consistency of the findings makes it unlikely that the excessive risks of death from lymphoma and pancreatic cancer are attributable to chance. An important aspect of the findings is that they provide a clue to the causation of cancer of the pancreas in man.

Many agents are known to increase the risk of lymphoma development in various species of laboratory and domestic animals. Several of them probably act indirectly by changing the immunological or hormonal status of animals or by interfering with some virus-host relationship in another way. There may well exist a similar variety of possible ways of increasing the risk to man of developing lymphoma. ${ }^{5-7}$

The results of further epidemiological studies and of detailed investigations of the occupational histories of individual chemists who develop cancer will be awaited with interest. In the meantime, greater awareness of cancer hazards is certainly needed in laboratories. It will be stimulated and guided by the publication of recommended codes of practice that refer to all known classes of chemical carcinogen. A proposal $^{8}$ to appoint safety officers, similar to radiation protection officers, with authority to see that chemical carcinogens are not used without adequate precautions deserves serious consideration.

\section{Headache and Hypertension}

For years doctors have disputed the association between headache and hypertension, but the public do not share the same doubts. They know that high blood pressure causes headache and that headache is an indication for a blood pressure check. Patients see an obvious cause-and-effect relationship between high pressure in the blood vessels and pain in the head. Many physicians agree. ${ }^{1}$ The typical hypertension headache is usually said to be throbbing in character and worst on waking in the morning, ${ }^{2}$ but headaches in hypertensive patients may have other explanations than the high blood pressure. Neurosis seems to be common among hypertensive patients. ${ }^{3}$ Neurotic people visit their doctors more often than non-neurotic people, and so their symptomless hypertension is more likely to be discovered. ${ }^{4}$ Headache may develop as a neurotic symptom after hypertension is discovered. 5 Few doctors would seriously regard high blood pressure as a result of psychic rather than renal disorder, but it has even been suggested that neurosis may be the cause of both the headache and the hypertension. ${ }^{6}$

Headache is a common affliction, and its apparent association with high blood pressure may be owed to its often being reported when patients are examined for hypertension. The relevance of the hypertension to the headache thus remains surprisingly blurred, and the report of a recent study carried out in Glasgow is welcome. ${ }^{7}$ In this study R. H. Al Badran and colleagues compared the incidence and type of headache in a group of hypertensive patients with those in a control group of normotensive patients. The study showed a steadily increasing incidence of headache in patients with diastolic pressures of $125 \mathrm{~mm}$. $\mathrm{Hg}$ or more, but there was no greater incidence of headache in patients with mild or moderate hypertension than in the control group. The headache in the severe hypertensives tended to recur daily on waking, it was shortlived, and it was poorly localized. Good control of blood pressure relieved the headache in most of these patients, but poor control did not usually do so.

By contrast with the severe hypertensives the milder hypertensives suffered headaches similar in incidence and character to those among the matched controls. They were often occipital and of longer duration but less frequent than in the severe hypertensives. In the normotensive controls and in patients with hypertension who failed to gain relief of headache despite good reduction of blood pressure cervical spondylosis was a commoner radiological finding than it was in the patients without headache or in those whose headache had been relieved by therapy.

1 Wolff, H. G., Headache, 2nd edn. London, Oxford University Press, 1963. Platt, R., British Medical fournal, 1950, 1, 951.

3 Pickering, G., High Blood Pressure, 2nd edn., p. 324. London, Churchill, 1968.

4 Robinson, J. O., Wood, M. M., British Fournal of Preventive and Social Medicine, 1968, 22, 23.

Stewart, I. McD. G., Lancet, 1953, 1, 1261.

7 Al Badran, R. H., Weir, R. J., and McGuiness, J. B., Scottish Medical fournal, 1970, 15, 48 .

${ }^{8}$ Pickering, G. W., Clinical Science, 1934, 1, 397. 
This establishment of a correlation between hypertension and headache only in severe hypertensives fits the physiology. Headache has a well-defined relationship with raised intracranial pressure from any cause. Increasingly severe headache is an almost constant prodromal symptom of hypertensive encephalopathy, in which the cerebrospinal-fluid pressure is invariably raised, and a raised intracranial pressure is frequently found in severe hypertension without any neurological symptoms other than headache. ${ }^{8}$

It seems therefore that headache, in so far as it is a symptom of hypertension, occurs only in severe cases and tends to disappear with effective reduction of blood pressure. Focal headaches in hypertensive patients, particularly when prolonged or persistent after effective treatment of the blood pressure, should direct the doctor's attention to other possible causes. These might be a berry aneurism, glaucoma, or even neurosis, and when headache is persistently occipital in location the possibility of cervical spondylosis should be considered-as it should also in normotensive patients in later life.

\section{Joints in Myxoedema}

Acromegaly, Cushing's disease, Addison's disease, and hyperparathyroidism" may all be associated with "rheumatic" symptoms, and recently two papers have appeared on the musculoskeletal features of hypothyroidism. ${ }^{2} 3$ This is no new discovery, for in $1873 \mathrm{Sir}$ William Gull" described "a cretinoid state" in five women with muscular stiffness, swollen joints, broad spade-like hands, and extensive infiltration of connective tissue. In $1877 \mathrm{Sir}$ William $\mathrm{Ord}^{5}$ reported five additional cases with many rheumatic symptoms and signs, including swollen, clumsy fingers, weak stiff muscles, lax ligaments, and joint effusions; he considered the symptoms due to swelling of the connective tissue throughout the body.

Recently D. N. Golding ${ }^{2}$ reported nine patients with myxoedema who presented with musculoskeletal symptomsgeneralized muscle and joint pains, muscle stiffness, cramps, and acroparaesthesiae; median nerve compression in the carpal tunnel was noted in three of the nine patients. In all cases symptoms were alleviated by administration of thyroxine. J. H. Bland and J. W. Frymoyer ${ }^{3}$ suspected hypothyroidism in 74 patients referred to the rheumatology unit in Vermont between 1954 and 1967. Laboratory studies confirmed the diagnosis in 38 patients: their paper described the findings in 11 patients in whom it was thought that the symptoms were due to hypothyroidism alone. The 11 patients studied had primary myxoedema. They all voluntarily mentioned stiffness, arthralgia, and swelling, and marked synovial thickening was present in all; ten had definite joint effusions. The knees and the small joints of the hands and feet were the most commonly affected joints. The synovial fluid was characterized by large volume, normal total protein concentration, normal cell count, and high viscosity. Radiographs showed osteoporosis in 9 of the 11 cases, and in three a peculiar destructive lesion was present, with apparent compression and crumbling of the

\footnotetext{
1 Bywaters, E. G. L., Dixon, A. St. J., and Scott, J. T., Annals of the Rheumatic Diseases, 1963, 22, 171.

Golding, D. N., Annals of the Rheumatic Diseases, 1970, 29, 10

Bland, J. H., and Frymoyer, J. W., New England fournal of Medicine, $1970,282,1171$

- Gull, W. W., Transactions of the Clinical Society of London, 1874, 7, 180.

- Ord, W. M., Medico-Chirurgical Transactions, 1878, 61, 57.
}

tibial plateau and marked loss of bone density, suggestive of a pathological fracture. The radiological appearances did not suggest gout or rheumatoid arthritis, nothing like rheumatoid erosions or tophi being seen.

It is of interest that in both series all patients made a complete and permanent recovery from their "rheumatic" symptoms on thyroid replacement therapy: in one of Golding's cases phenylbutazone, which is known to have in some instances an antithyroid action, made the symptoms worse. None of the cases in the American series had previously been diagnosed as suffering from hypothyroidism, and only one of Golding's patients looked typically myxoedematous. Here, then, is another arthropathy which, if diagnosed, is eminently and satisfactorily treatable.

\section{What about the Patient?}

If anything certain has emerged from the discussions over the past year or so about the role of the general practitioner in the hospital it is that a variety of roles are possible, not merely one. This week three doctors talk about them in our "Contemporary Themes" section. A tape-recorded discussion (page 335) between a general practitioner, a medical officer of a regional hospital board, and a consultant physician is centred on the theme, "Has the G.P. a Place in the Hospital?" Some doubt surrounds the future development of the hospital services in this country-a perfectly laudable doubt, for large sums must be invested in a singularly unpredictable future. But, if the technocratic view of a hospital as a place for treatment to the immediately profitable limit and then instant discharge is carried to its logical conclusion, we may be asking one day, "Has the patient a place there either?"

In earlier times a hospital was a place where the poor and needy found refuge, often at the expense of a charity. "A place of rest and entertainment" is the O.E.D.'s first definition, and perhaps the television set in the ward keeps up that tradition. Today the hospital planners are apt to forget that though we see a different kind of "poverty" and "need" around us there are many people who still require the older kind of hospital care. Fortunately the cure-or-discharge people with their slide-rules and bed-occupancy rates have not yet succeeded in driving out all compassion. In fact their statistics can be helpful provided they are not taken too seriously.

But are there patients whose needs are better satisfied by being under their general practitioner than under a consultant when they are in a hospital bed? Many people would say that there are, though the question seems to have been rarely studied outside the field of obstetrics. What we hear much more about in debates on the general practitioner in hospital is whether it will halt emigration, provide an educative experience, be adequately paid, upset the running of the hospital, be more economical of the nation's resources-all important issues. But a thorough study of the needs of patients in relation to the care that general practitioners could provide must throw light from another angle on the matter. Much too might be learnt from countries like Canada where general practitioners already have a substantial part in running the hospitals. The speakers in this week's discussion argue refreshingly on "Admission for Assessment," which is one topic on which some precise facts and figures might be worth studying. 